\title{
A search for OB stars in the field of the galactic OB association Bochum 7^
}

\section{UBV-IR photometry and optical spectroscopy}

\author{
M. Corti ${ }^{1}$, G. Bosch ${ }^{1}$, and V. Niemela ${ }^{2}$ \\ 1 Facultad de Ciencias Astronómicas y Geofísicas, U.N. La Plata, Paseo del Bosque s/n, 1900 La Plata, Argentina, Instituto de \\ Astrofísica de La Plata (IALP), CONICET, Argentina \\ e-mail: mariela@lilen.fcaglp.unlp.edu.ar \\ 2 Member of Carrera del Investigador Científico, CIC-BA, Visiting Astronomer, CTIO, NOAO, operated by AURA, Inc., for NSF \\ Facultad de Ciencias Astronómicas y Geofísicas, U.N. La Plata, Paseo del Bosque s/n, 1900 La Plata, Argentina
}

Received 17 Febuary 2006 / Accepted 15 January 2007

\begin{abstract}
Context. The presence of a Wolf-Rayet star, WR12, at $\alpha=8^{\mathrm{h}} 44^{\mathrm{m}} 47.2^{\mathrm{s}}, \delta=-45^{\circ} 58^{\prime} 55.5^{\prime \prime}(\mathrm{J} 2000.0)$, triggered the search for a surrounding OB association now known as Bochum 7. Several papers have dealt with the existence of Bo 7, but none actually included spectral types of the stars. To assure the spectral types of members of the association, it is essential to have spectral images of them. Aims. In this paper we present previously unpublished spectra of OB stars over a 30 arcmin field covering the estimated field of the galactic OB association.

Methods. From UBV aperture photometry, OB star candidates were selected, and optical spectroscopic observations were obtained for these in CASLEO, Argentina.

Results. 63 stars were classified as OB type, including 8 stars already present in the LSS catalogue, and 37 of these 63 stars did not have a well-known spectral type. The OB stars were used to test the properties of the interstellar dust in the line of sight and the relation between the diffuse interestelar band at $4428 \AA$, which grows linearly with $E_{(B-V)}$ until 1 mag. Combining our data with 2MASS IR photometry we tested and confirmed that derivation of stellar parameters from our photometry, using the CHORIZOS code, is highly reliable.
\end{abstract}

Key words. Galaxy: open clusters and associations: individual: Bochum 7 - stars: early-type - techniques: spectroscopic techniques: photometric

\section{Introduction}

Relatively low extinction at the region in the third quadrant in the Galactic plane allows deep analysis of the stellar population and galactic structure towards the outskirts of our Galaxy. The lack of tightly bound and easily identifiable young open clusters leads to the quest for OB associations as tracers of spiral arms. Massive stars are usually found in groups, and the presence of a Wolf-Rayet star, WR12 in the catalogue of galactic WR stars (van der Hucht 2001), at $\alpha=8^{\mathrm{h}} 44^{\mathrm{m}} 47.2^{\mathrm{s}}, \delta=-45^{\circ} 58^{\prime} 55.5^{\prime \prime}$ (J2000.0), triggered the search for a surrounding OB association. Moffat \& Vogt (1975) suggested that several OB stars listed in the LSS catalogue (Stephenson \& Sanduleak 1971) around WR12 may be an OB association, namely Bochum 7, at $5.8 \mathrm{kpc}$, although the membership of WR12 itself was in doubt. Lundstrom \& Stenholm (1984) proposed that Bo 7 is an OB association of $\sim 30 \mathrm{pc}$ of size. On the other hand, Sung et al. (1999) suggested that the stars associated with Bo 7 are only a local concentration in the density of young stars belonging to the Vel OB3 association. From data of the 7 stars listed in the LSS catalogue, Corti et al. (2003) derive a spectroscopic and kinematical distance of about $5.0 \mathrm{kpc}$ for Bo 7, supporting the idea

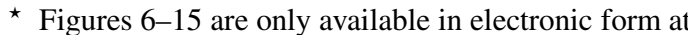
http://www. aanda.org that the Wolf-Rayet star LS $1145=$ WR 12 was also member of this association.

In this work, we present the analysis made with photometric and spectroscopic data of the stars in a 30 arcmin field surrounding WR 12. With these we obtained spectral types, colour excesses, equivalent widths of the DIB 4428 feature, and distances for the OB stars found in the observed field. By comparing stellar temperatures and reddening derived from photometric and spectroscopic data we analyse the reliability of the (more abundant) photometric ones.

The paper is organized as follows: in Sect. 2 we describe the observations. In Sect. 3 we present the results and their discussion, and in Sect. 4 we summarize the main issues. In a later paper (Paper II) we will increase the number of stars that will be analysed, including OB stars without spectral data. We will estimate the distances and colour excesses of other possible members. Combining this information with proper motions we will be able to identify the stellar groups and to infer their evolutionary stages.

\section{Observations}

\subsection{Photometric data}

We obtained $U B V$ photometric images with the 1-m CurtisSchmidt telescope and a Thompson $1024 \times 1024$ CCD 
with $19 \mu \mathrm{m}$ pixels at the Cerro Tololo Inter-American Observatory (CTIO), Chile, in March 5, 1994. This instrumental configuration yields a $30 \times 30$ arcmin field of view, centred at $\alpha=8^{\mathrm{h}} 44^{\mathrm{m}} 23^{\mathrm{s}}, \delta=-45^{\circ} 56^{\prime} 22^{\prime \prime}(\mathrm{J} 2000.0)\left(l=265^{\circ} .12\right.$, $\left.b=-2^{\circ}\right)$. The scale on the detector is $1.834 \mathrm{arcsec} /$ pixel, which subsamples a stellar image under average seeing conditions at CTIO. CCD acquisition was set to obtain images with GAIN $=3.3 \mathrm{e}^{-} /$ADU and RDNOISE $=7 \mathrm{e}^{-}$.

Different exposure times were used for each filter: $10 \mathrm{~s}$ for the $V$ and $B$ filters and $20 \mathrm{~s}$ for the $U$ filter. The same number of images were taken for the Landolt photometric standard region sa104 (Landolt 1992). For these images the exposure times were 10,20 , and $60 \mathrm{~s}$ for the $V, B$, and $U$ filters respectively. The transformation relations used here were:

fit $u 1=0.0, u 3=0.000$

const $\quad u 4=-0.022, u 2=0.591, u 5=-0.023$

$$
\begin{aligned}
m U= & (U B+B V+V)+u 1+u 2 \cdot X U+u 3 \cdot U B \\
& +u 4 \cdot U B \cdot X U+u 5 \cdot B V \cdot X U
\end{aligned}
$$

fit $\quad b 1=0.0, b 3=0.000$

const $\quad b 4=-0.023, b 2=0.276$

$$
\begin{aligned}
m B= & (B V+V)+b 1+b 2 \cdot X B+b 3 \cdot B V \\
& +b 4 \cdot B V \cdot X B
\end{aligned}
$$

fit $\quad v 1=0.0, v 3=0.000$

const $\quad v 4=0.007, v 2=0.152$

$m V=V+v 1+v 2 \cdot X V+v 3 \cdot B V+v 4 \cdot B V \cdot X V$,

where $m U, m B$, and $m V$ are the instrumental magnitudes; $U B$ and $B V$ are the colour indices; $V$ is the visual magnitude; and $X U$, $X B$, and $X V$ are the air masses of each filter. We also downloaded near-infrared $J H K$ magnitudes for stars within our observed area from the Two Micron All Sky Survey (2MASS) Point Source Catalog (Cutri et al. 2003).

\subsection{Digital spectra}

Spectral images were obtained at the Complejo Astronómico El Leoncito (CASLEO) ${ }^{1}$, San Juan, Argentina, during several observing runs between 2000 and 2002. The instrumental configurations used were: the Boller and Chivens (B\&C) and the REOSC Cassegrain spectrographs attached to the 2.15-m telescope at CASLEO. In both spectrographs we used a $6001 \mathrm{~mm}^{-1}$ grating, and the slit width was set to match an aperture of 2 to 3 arcsec according to the average seeing at CASLEO. Fifteen spectra were secured with the B\&C spectrograph during January 2001. This spectrograph holds a Photometrics CCD of $512 \times 512$ pixels, at a $20 \mu \mathrm{m}$ pixel size. The reciprocal dispersion of these spectra is $\simeq 2.3 \AA \mathrm{px}^{-1}$ and the spectral range $3800-5000 \AA$. The signal-to-noise ratio was always higher than 120 . One hundred and eighty spectra were obtained with the REOSC Cassegrain spectrograph during January 2000, February 2001, and January and February 2002,

\footnotetext{
1 Operated under agreement between CONICET, SeCyT and the National Universities of La Plata, Córdoba and San Juan, Argentina.
}

using a TEK $1024 \times 1024$ pixel CCD detector, with a pixel size of $24 \mu \mathrm{m}$. The reciprocal dispersion was $\simeq 1.65 \AA \mathrm{px}^{-1}$. The spectral range of these spectra is $3700-5200 \AA$ and the signal-tonoise ratio ranges from 150 to 200 .

He-Ar comparison lamp images were observed at the same telescope position as the stellar images immediately after or before the stellar exposures. Bias frames were also obtained every night, as well as spectra of the planetary nebula IC418 and HR 1996 as radial velocity standards stars. All spectra were processed and analysed with IRAF $^{2}$ routines at La Plata Observatory.

\section{Results and their discussion}

\subsection{Photometry}

IRAF's standard image reduction procedures were followed and aperture photometry was calculated on science and standard images, as subsampling prevented us from performing point-spread function photometry. The aperture radius chosen was 1.5 pixels, and the sky annulus inner radius was 5 pixels from the star centre and 3 pixels wide. Approximately 2000 stars were identified in the field of view including stars as faint as $V \sim 18$.

We compared our photometric results with those of Sung et al. (1999), who had obtained $U B V I$ CCD photometry for the region centred at $\alpha=8^{\mathrm{h}} 44^{\mathrm{m}} 47.2^{\mathrm{s}}, \delta=-45^{\circ} 58^{\prime} .9$ (J2000.0) using the $1-\mathrm{m}$ telescope (f/8) at Siding Spring Observatory. To do so, we first derived an astrometric calibration for our stars, using the ASTROM task within STARLINK software package. We then correlated our photometry table with that of Sung et al. (1999), matching stars by their celestial coordinates. In this way, we found $\simeq 1100$ stars in common, with more than 300 stars with magnitudes brighter than 15 in the $V$-band. The comparison between both photometries can be seen in Fig. 2, where it is possible to detect a minor systematic difference $(0.04 \mathrm{mag})$ between our photometry and that of Sung et al. (1999). We have also used their photometry as reference for estimating an upper limit for the overall uncertainty in our derived magnitudes, obtaining the magnitude differences between our photometry and that of Sung et al. (1999), which was found to be about 0.08 mag in $V$, $0.10 \mathrm{mag}$ in $B$, and $0.18 \mathrm{mag}$ in $U$. This way, the uncertainty in our colours were $0.13 \mathrm{mag}$ in $(B-V)$ and $0.21 \mathrm{mag}$ in $(U-B)$. The colours $(B-V)$ and $(U-B)$ and the magnitudes $V$ are listed in Table 1.

We analysed our $U B V$ photometric data and selected more than 100 stars brighter than $V=15$ with colours belonging to early-type stars. The selection was done using the reddeningfree parameter $Q$ (Schmidt-Kaler 1982). Although it would be safer to pick only stars with $Q<-0.5$ as early-type candidates, the number of stars with $Q$ satisfying this and also brighter than 15 mag was too small. We therefore included stars with $-0.5<Q<-0.3$, although the probability of detecting true B-type stars was unavoidably reduced. This brightness limit was included to build our target list for the spectroscopic runs described in Sect. 2.2 as the need of high signal-to-noise spectra imposes further restrictions on the target magnitudes for obtaining spectra at CASLEO.

\footnotetext{
2 IRAF is distributed by NOAO, operated by AURA, Inc. under an agreement with NSF.
} 
Table 1. Photometric and spectroscopic information for individual stars analysed in this work.

\begin{tabular}{|c|c|c|c|c|c|c|c|}
\hline$\overline{\overline{I D}}$ & $\overline{\bar{V}}$ & $\overline{(B-V)}$ & $\overline{(U-B)}$ & $\overline{\text { SpT }}$ & eqw4428 & $\overline{V_{\circ}-M_{v}}$ & Cross ref. \\
\hline CBN84332.2-455337 & 10.1 & 0.30 & -0.41 & B2IV & 0.60 & 11.1 & LS1132 \\
\hline CBN84433.4-454115 & 10.4 & 0.23 & -0.47 & B2III & 0.85 & 12.5 & 2MASS 8443341-4541167 \\
\hline CBN84501.9-455034 & 10.4 & 0.18 & -0.45 & B2IV & 0.73 & 11.9 & CD-454491 \\
\hline CBN84446.2-460433 & 10.7 & 0.08 & -0.49 & B3V & 0.60 & 11.2 & CD-454481 \\
\hline CBN84324.0-460831 & 10.8 & 0.50 & -0.50 & $07.5 \mathrm{~V}$ & 1.90 & 12.8 & $\mathrm{LS}_{1131^{\wedge}}$ \\
\hline CBN84447.2-455856 & 10.8 & 0.51 & -0.36 & WN8 ${ }^{\diamond}$ & $\ldots$ & $\ldots$ & LS1145 \\
\hline CBN84506.0-455008 & 10.9 & 0.25 & -0.35 & $\mathrm{~B} 2 \mathrm{~V}$ & 0.60 & 11.3 & CD-454493 \\
\hline CBN84349.6-460711 & 10.9 & 0.34 & -0.61 & $\mathrm{O} 6.5 \mathrm{~V}((\mathrm{f}))$ & 1.60 & 13.5 & LS1135 \\
\hline CBN84447.4-460339 & 11.1 & 0.09 & -0.39 & B3-5V & 0.70 & 11.4 & CD-454483 \\
\hline CBN84510.4-455854 & 11.2 & 0.55 & -0.48 & O9.5II & 2.50 & 13.8 & CPD-452977 \\
\hline CBN84504.8-460013 & 11.2 & 0.14 & -0.44 & $\mathrm{~B} 2 \mathrm{~V}$ & 0.70 & 12.2 & CD-454492 \\
\hline CBN84406.6-455642 & 11.3 & 0.17 & -0.32 & $\mathrm{~B} 2 \mathrm{~V}$ & 0.80 & 12.2 & CD-454466? \\
\hline CBN84424.9-455334 & 11.3 & 0.61 & -0.44 & $07.5 \mathrm{~V}$ & 1.90 & 12.9 & LS1144` \\
\hline CBN84426.0-455749 & 11.4 & 0.25 & -0.16 & B8V & 0.60 & 10.3 & CD-454473 \\
\hline CBN84450.1-455648 & 11.4 & 0.16 & -0.51 & $\mathrm{~B} 2 \mathrm{~V}$ & 0.80 & 12.3 & CD-454484 \\
\hline CBN84350.9-460348 & 11.4 & 0.45 & -0.49 & $09-9.5 \mathrm{~V}$ & 1.90 & 13.0 & LS1137 \\
\hline CBN84506.3-460906 & 11.5 & 0.55 & -0.47 & B0IIIe & 1.90 & 12.5: & LS1147 \\
\hline CBN84543.1-460742 & 11.5 & 0.10 & -0.42 & B2-3V & 0.40 & 12.4 & CPD-453000 \\
\hline CBN84450.9-461012 & 11.6 & 0.47 & -0.48 & B1III & 2.38 & 13.4 & LS1146“ \\
\hline CBN84404.4-455316 & 11.7 & 0.68 & -0.33 & B0III & 2.27 & 13.2 & LS1140 \\
\hline CBN84339.5-455148 & 11.7 & 0.54 & -0.14 & B2III & 0.74 & 12.9 & CPD-452909 \\
\hline CBN84518.6-454543 & 11.8 & 0.27 & -0.30 & B2V & 0.48 & 12.4 & CPD-452984 \\
\hline CBN84325.3-460750 & 11.8 & 0.25 & -0.05 & B8-A0V & 0.40 & 10.4 & CPD-452901 \\
\hline CBN84552.3-460929 & 11.8 & 0.10 & -0.41 & B5V & 0.44 & 12.1 & \\
\hline CBN84359.6-460237 & 12.0 & 0.20 & -0.28 & B5-8V & 0.70 & 11.5 & CPD-452926 \\
\hline CBN84338.5-460817 & 12.0 & 0.45 & -0.52 & $09.5 \mathrm{~V}$ & 1.90 & 13.5 & CPD-452910 \\
\hline CBN84349.4-454204 & 12.0 & 0.29 & -0.25 & B3-5V & $\ldots$ & 11.7 & CPD-452917 \\
\hline CBN84443.0-455130 & 12.0 & 0.21 & -0.28 & B8V & 0.50 & 11.0 & CPD-452953 \\
\hline CBN84318.7-460742 & 12.2 & 0.24 & -0.19 & B8V & $\ldots$ & 11.1 & CPD-452895 \\
\hline CBN84438.9-460746 & 12.2 & 0.10 & -0.36 & B5V & 0.50 & 12.4 & 2MASS $8443908-4607450$ \\
\hline CBN84327.7-460929 & 12.3 & 0.63 & -0.58 & B0V-III & 2.20 & 13.4 & 2MASS 8432796-4609263 \\
\hline CBN84348.6-460736 & 12.3 & 0.40 & -0.47 & B1-1.5V & 1.70 & 12.9 & 2MASS 8434879-4607337 \\
\hline CBN84434.3-455214 & 12.5 & 0.23 & -0.30 & B5V & 1.40 & 12.3 & 2MASS 8443427-4552136 \\
\hline CBN84505.0-460300 & 12.6 & 0.16 & -0.37 & B2.5-3V & 0.40 & 12.9 & 2MASS 8450512-4602598 \\
\hline CBN84446.7-454632 & 12.6 & 0.46 & -0.09 & B3-5V & 0.40 & 11.8 & 2MASS 8444674-4546333 \\
\hline CBN84327.4-460240 & 12.6 & 0.56 & -0.44 & B0V & 1.60 & 13.4 & 2MASS 8432763-4602382 \\
\hline CBN84500.4-455834 & 12.6 & 0.24 & -0.34 & B5V & 0.70 & 12.3 & 2MASS $8450044-4558343$ \\
\hline CBN84505.7-454040 & 12.7 & 0.60 & -0.31 & B1III & 2.60 & 14.0 & 2MASS $8450573-4540420$ \\
\hline CBN84526.1-460527 & 12.9 & 0.66 & -0.09 & B2III & 2.10 & 13.8 & 2MASS 8452620-4605258 \\
\hline CBN84350.7-455820 & 12.9 & 0.55 & -0.34 & $\mathrm{~B} 1.5 \mathrm{~V}$ & 2.60 & 12.5 & 2MASS 8435084-4558194 \\
\hline CBN84344.7-460656 & 12.9 & 0.43 & -0.44 & B1V & 1.90 & 13.5 & 2MASS 8434493-4606537 \\
\hline CBN84522.4-455527 & 13.0 & 0.72 & -0.24 & B1III & 2.60 & 13.9 & 2MASS 8452244-4555280 \\
\hline CBN84502.0-455257 & 13.2 & 0.23 & -0.11 & B8-A0V & 0.70 & 11.8 & 2MASS 8450219-4552572 \\
\hline CBN84410.6-455452 & 13.3 & 0.37 & -0.08 & B5-8V & 0.80 & 12.2 & 2MASS 8441074-4554522 \\
\hline CBN84419.9-455552 & 13.5 & 0.65 & -0.08 & B2III & 2.90 & 14.3 & 2MASS 8441999-4555524 \\
\hline CBN84458.5-455242 & 13.5 & 0.24 & -0.11 & B8-9V & & 12.3 & 2MASS 8445857-4552434 \\
\hline CBN84431.5-455314 & 13.5 & 0.61 & -0.34 & B2III: & 2.36: & 14.3: & 2MASS $8443163-4553148$ \\
\hline CBN84518.2-455816 & 13.6 & 0.65 & -0.32 & B $0.5 \mathrm{~V}$ & 3.00 & 14.1 & 2MASS 8451822-4558166 \\
\hline CBN84423.1-460821 & 13.7 & 0.65 & -0.33 & B1-2/V-III & 1.80 & 14.0 & \\
\hline CBN84325.6-460820 & 13.7 & 0.62 & -0.34 & $\mathrm{~B} 2 \mathrm{Ve}$ & 2.00 & 14.5: & 2MASS 8432574-4608174 \\
\hline CBN84421.9-455136 & 13.7 & 0.66 & -0.24 & B1V & 3.48 & 13.9 & \\
\hline CBN84437.8-455257 & 13.8 & 0.43 & -0.32 & B8-A0V & 0.30 & 11.8 & 2MASS 8443788-4555288 \\
\hline CBN84510.9-455900 & 14.0 & 0.61 & -0.31 & B1V & 2.32 & 14.2 & \\
\hline CBN84442.0-460802 & 14.1 & 0.51 & -0.14 & B2V & 1.10 & 14.0 & 2MASS 8444210-4608004 \\
\hline CBN84346.7-460641 & 14.2 & 0.37 & -0.43 & B1-5V & 2.20 & 14.2 & 2MASS 8434686-4606390 \\
\hline CBN84430.9-455442 & 14.2 & 0.53 & -0.30 & $\mathrm{~B} 2 \mathrm{~V}$ & 1.77 & 14.1 & \\
\hline CBN84523.5-455340 & 14.3 & 0.51 & -0.06 & B8V & 1.80 & 12.4 & 2MASS 8452359-4553411 \\
\hline CBN84416.7-461121 & 14.3 & 0.53 & -0.25 & $\mathrm{~B} 1.5 \mathrm{~V}$ & 1.90 & 14.2 & 2MASS 8441683-4611189 \\
\hline
\end{tabular}

\subsection{Spectroscopy}

\subsubsection{Spectral types}

The spectral classification of selected stars was done by comparing obtained spectra with the Digital Atlas of OB Stars published by Walborn \& Fitzpatrick(1990, WF90). As the spectral resolution of our data $\left(1.65 \AA \mathrm{px}^{-1}\right)$ was lower than the WF90 atlas $\left(0.5 \AA \mathrm{px}^{-1}\right)$, we have also obtained high signal-to-noise spectra $(S / N \geq 200)$ of the atlas stars to detect important features for the classification of early-type 
Table 1. continued.

\begin{tabular}{cccclcll}
\hline \hline ID & $V$ & $(B-V)$ & $(U-B)$ & SpT & eqw4428 & $V_{\circ}-M_{v}$ & Cross ref. \\
\hline CBN84423.2-455337 & 14.5 & 0.58 & -0.24 & B1.5-2V & 1.15 & 14.1 & 2MASS 8442335-4553371 \\
CBN84418.2-455307 & 14.6 & 0.56 & -0.32 & B1V & 1.30 & 14.7 & 2MASS 8441838-4553078 \\
CBN84414.0-455733 & 14.7 & 0.76 & -0.16 & B1.5-2V & 1.90 & 13.9 & 2MASS 8441413-4557329 \\
CBN84421.9-460125 & 14.8 & 0.49 & -0.27 & B1V & 2.00 & 15.2 & 2MASS 8442208-4610224 \\
CBN84518.8-454147 & 14.8 & 0.57 & -0.13 & B5V & 0.87 & 13.3 & 2MASS 8451888-4541494 \\
CBN84527.2-455360 & 14.9 & 0.49 & +0.01 & B5-8V & 1.14 & 13.5 & 2MASS 8452725-4554006 \\
\hline
\end{tabular}

$\diamond$ van der Hucht (2001).

Column 1 lists the stars' identification according to the guidelines of the IAU, i.e., using the (capitalized) first letters of the last names of the authors and the numbers, of the celestial coordinates in the J2000.0 frame. Columns 2 to 4 list the UBV magnitude and colours; the MK spectral classification is listed in Col. 5. The $\lambda 4428$ equivalent width is showed in $\AA$ in Col. 6. Column 7 shows the spectrophotometric distance modulus determined adopting the absolute magnitudes corresponding to the spectral types, according to the calibration by Martins et al. (2005) for O spectral types and Schmidt-Kaler (1982) for B spectral types. Column 8 includes the cross reference for these stars, where LS indicates stars in the Luminous Star Catalogue (Stephenson \& Sanduleak 1971), CD indicates stars in the Córdoba Durchmusterung catalogue, CPD indicates stars in the Cape Photographic Durchmusterung catalogue, and 2MASS indicates stars in the 2MASS catalogue (Cutri et al. 2003).

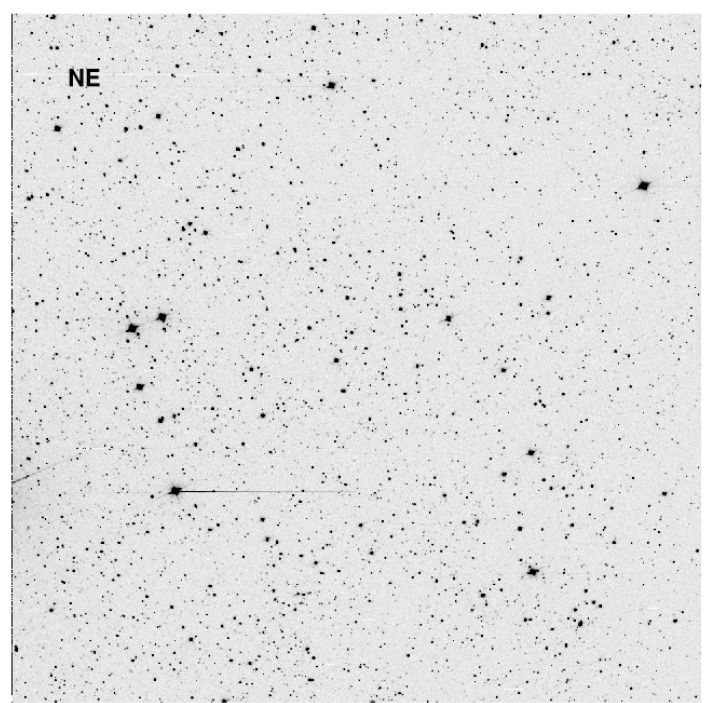

Fig. 1. $V$ frame of Bochum 7 centred at $\alpha=8^{\mathrm{h}} 44^{\mathrm{m}} 47.2^{\mathrm{s}}, \delta=-45^{\circ} 58^{\prime} .9$ (J2000.0). The scale on the detector is 1.834 arcsec/pixels and the field covered is 30 arcmin on each side.

stars. However, the signal-to-noise ratio decreases for fainter stars, leading to somewhat larger uncertainties in the spectral classification. With these data we are reproducing the WF90 atlas for lower dispersion spectrographs. A preliminary version of the atlas is already available on the internet at http://fcaglp.unlp.edu.ar/ mariela/OBSpectralAtlas.

From a final set of 102 stars, we classified 63 stars as early (OB) type, among which 50 are main sequence objects and 13 are subgiants, giants, or luminous giants. Among these 63 OB type stars, we found new spectral types for 37 stars. From Fig. 4 to Fig. 15 show the complete sample of classified spectra. The complete list can be found in Table 1. The availability of our Atlas of OB standards allowed us to reassess previous spectral classification (Corti et al. 2003). In this way, we classified LS 1132 as a B2IV and LS 1147 as a B0IIIe, previously classified as B3V and B1 Ve, respectively. We searched the data about the spectral types of these 63 stars in the "Catalog of Galactic OB Stars" (Reed 2003), and we only found our own references (Corti et al. 2003) for the stars in the Luminous Star Catalogue (Stephenson \& Sanduleak 1971), together with SpT = O6III (Vijapurkar \& Drilling 1993) and O7Vn (Vijapurkar \& Drilling 1993) for LS 1135 and LS 1131, respectively.

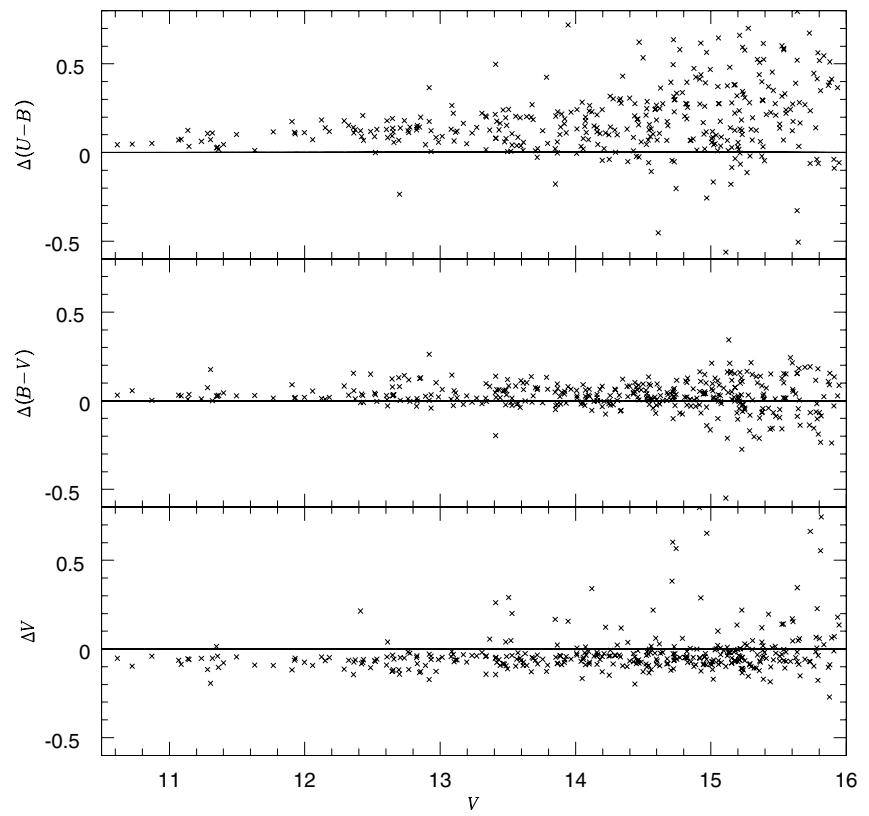

Fig. 2. Differences between our photometry and that of Sung et al. (1999). There is a minor difference between both photometries of about $0.04 \mathrm{mag}$ in $V$. The lines for 0 mag and 0 colours show the offsets more clearly.

With our spectral classification we are able to test the derivation of basic stellar parameters, such as temperature and observed extinction, from photometric data. This will be useful when analysing the larger sample of stars for which we only have photometry available for the $U B V J H K_{\mathrm{s}}$ analysis and for which we employed the CHORIZOS code (Maíz-Apellániz 2004). For this we used the atmospheres of Kurucz (2004) with $Z=0.0$ and main sequence gravity as model atmospheres. The Kurucz atmospheres were extinguished using $R_{5495}=2.0-6.0+$ MC laws and $E(4405-5495)$ ranging from -0.5 to 5.0. The CHORIZOS code did the zero point (hereafter ZP) correction in all the synthetic magnitudes, employing the ZP correction of Maíz Apellániz (2006) for the Johnson system $U B V$ and the ZP correction of Cohen et al. (2003) for the 2MASS $J H K_{\mathrm{s}}$ photometry. Figure 3 shows the comparison of the star's temperature obtained with the spectral classification and that obtained with the photometric data. An overall good agreement, within observational errors, can be readily see. Stars with notorious discrepancies are labelled in the plot and discussed below. Among the hotter stars, 


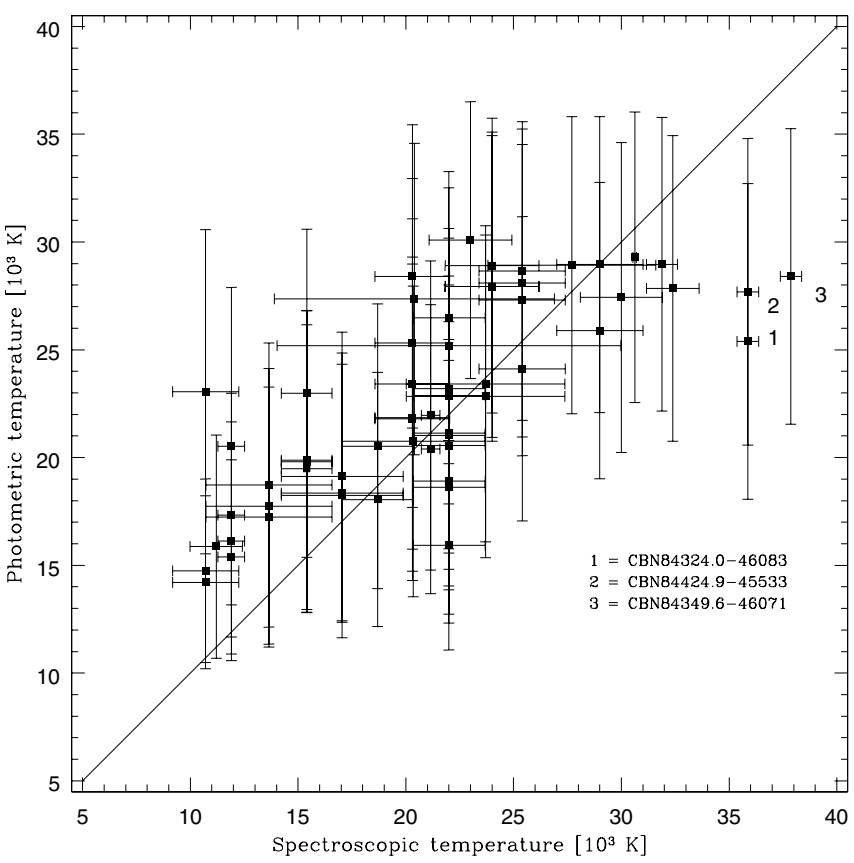

Fig. 3. Relation between the temperature values obtained from our photometric data using the CHORIZOS code ( $y$-axis) and the data derived from known spectral types ( $x$-axis). CBN84349.6-460711 is a binary system and CBN84324.0-460831 and CBN84424.9-455334 are two real candidates of binary systems. See discussion in the text.

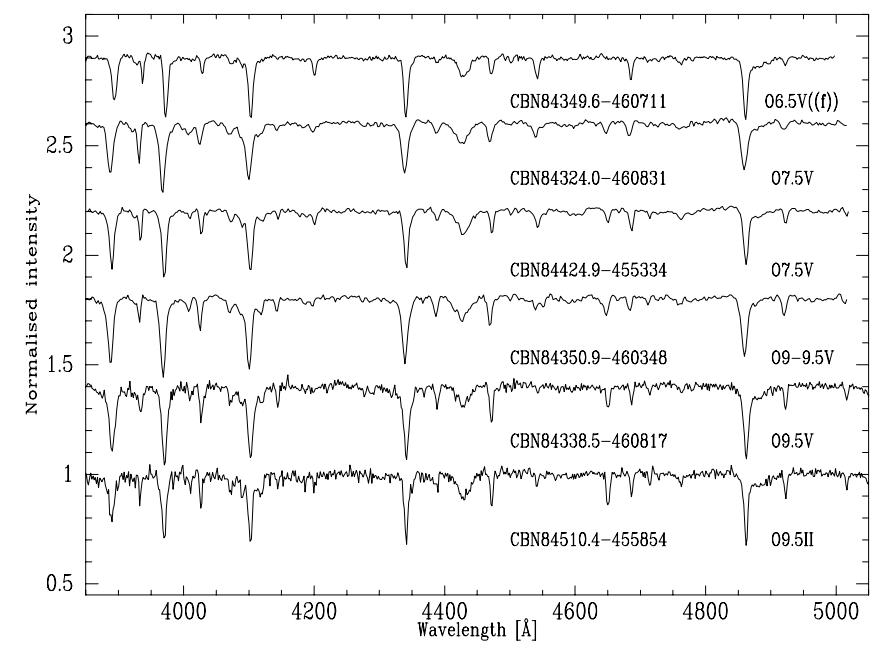

Fig. 4. Sample spectra of stars in the region of Bochum 7. Spectra are normalised. This figure includes a wide range of $\mathrm{O}$ spectral types. The complete list can be found in Table 1.

CBN84349.6-460711 is a binary system (Corti et al. 2003) and CBN84324.0-460831 and CBN84424.9-455334 are two strong candidates of binary system (Corti 2005). The last two stars show large variations among the radial velocities at the absortion lines in their respective spectra (Corti, in preparation). The reason for the discrepancy between the temperature obtained with the spectral classification and photometric data of these stars could be that the secondary component participates in their colours and for this reason the temperature obtained with the photometric data does not agree with the spectroscopic temperature.

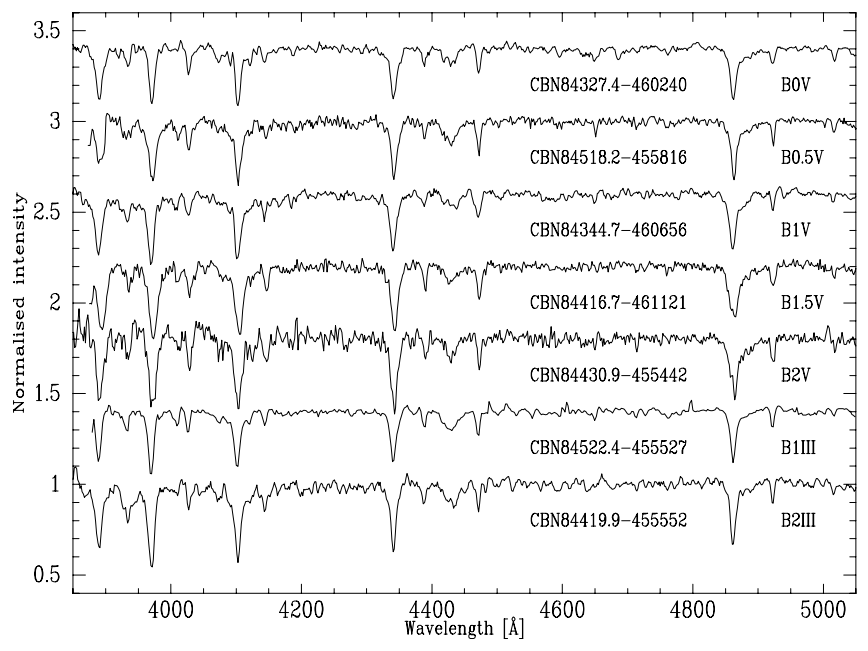

Fig. 5. Sample spectra of stars in the region of Bochum 7. Spectra are normalised. This figure includes a wide range of B spectral types. The complete list can be found in Table 1 .

\subsection{2. $4428 \AA ̊$ diffuse interstellar band (DIB)}

We studied the $4428 \AA$ DIB in our medium resolution spectra of OB stars. We fitted a Gaussian function to the profile of the DIB in 6 O-type and 54 B-type stars of our sample, and measured the equivalent width (EW) of the DIB in the spectrum of each star. EWs are listed in Table 1. This DIB is well evident in the blue optical spectra in Fig. 4. Stars of early spectral type, such as those included in this work, are especially useful for $4428 \AA$ diffuse band studies.

Figure 16 shows the correlation of $\lambda 4428$ equivalent width with the stellar colour excess $E_{(B-V)}$, for the sample shown in Fig. 1. We have added data points from Herbig (1975) so as to include DIB measurements for stars of the same reddening as those in our sample. We have also added points from Snow et al. (2002) representing very heavily reddened lines of sight in Cygnus. The plot shows a positive correlation, as suggested by Herbig (1975), at least up to roughly $E_{(B-V)} \simeq 1.0 \mathrm{mag}$ in our data. However, we find a steeper slope for the linear fit through our data than Herbig (1975), and it can also be seen that our data seem to complement those of Snow et al. (2002) better.

\subsection{Interstellar extinction}

The combination of photometric and spectroscopic information provides the only chance of determining individual colour excesses of the program stars, and hence of analysing the behaviour of the interstellar extinction in the line of sight. Figure 17 shows the photometric $E_{(B-V)}$ obtained using the CHORIZOS code with the atmosphere models mentioned previously vs. spectroscopic $E_{(B-V)}$, both with their error bars. It is possible to see a very good correspondence between both.

In the colour-colour diagram (Fig. 18), we have plotted the sixty-three OB stars and the other thirty-nine stars with spectral types between A0 and G3. It can be seen that the contamination of stars earlier than B8 type increases sharply as we move down to A0 type stars (Schmidt-Kaler 1982). There are two stars named CBN84431.8-455813 and CBN84446.0-45544 that do not fall where expected according to their spectral types. We reviewed their photometry and their spectral data and could not find any visible source for this odd behaviour, although they could be unresolved visual binaries. 


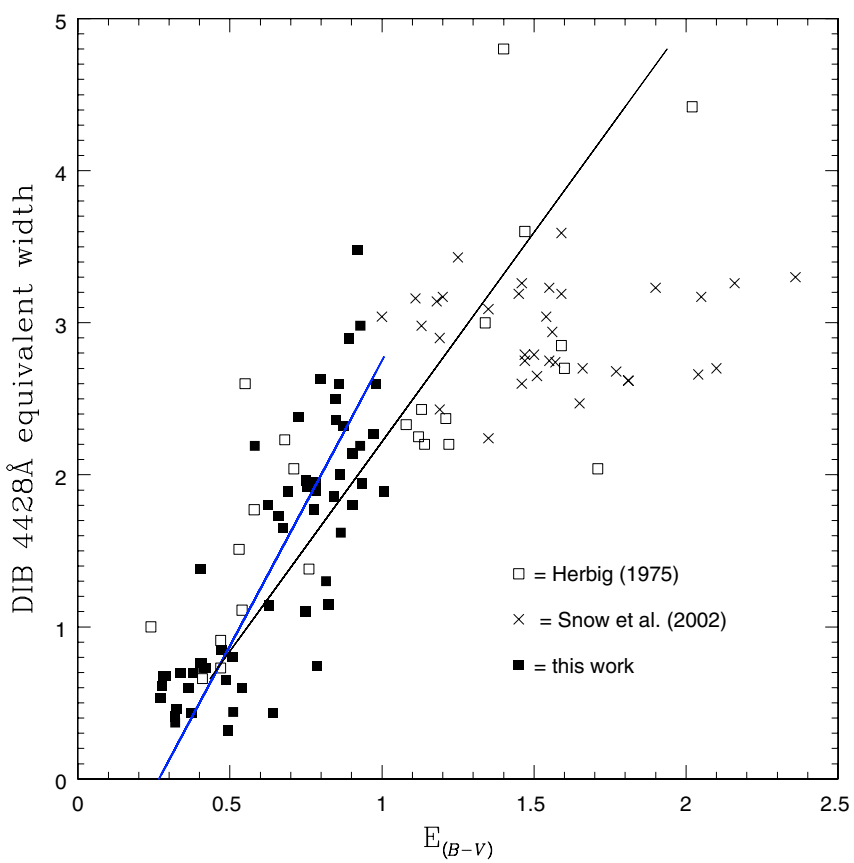

Fig. 16. Correlation of $\lambda 4428$ equivalent width with colour excess $E_{(B-V)}$. The open squares are data from Herbig (1975), the crosses are data from Snow et al. (2002), and the full squares are our own data. Within the broad scatter evident in this diagram, we see that the equivalent width of the $4428 \AA$ DIB levels off with increasing extinction beyond $E_{(B-V)} \simeq 1.0 \mathrm{mag}$.

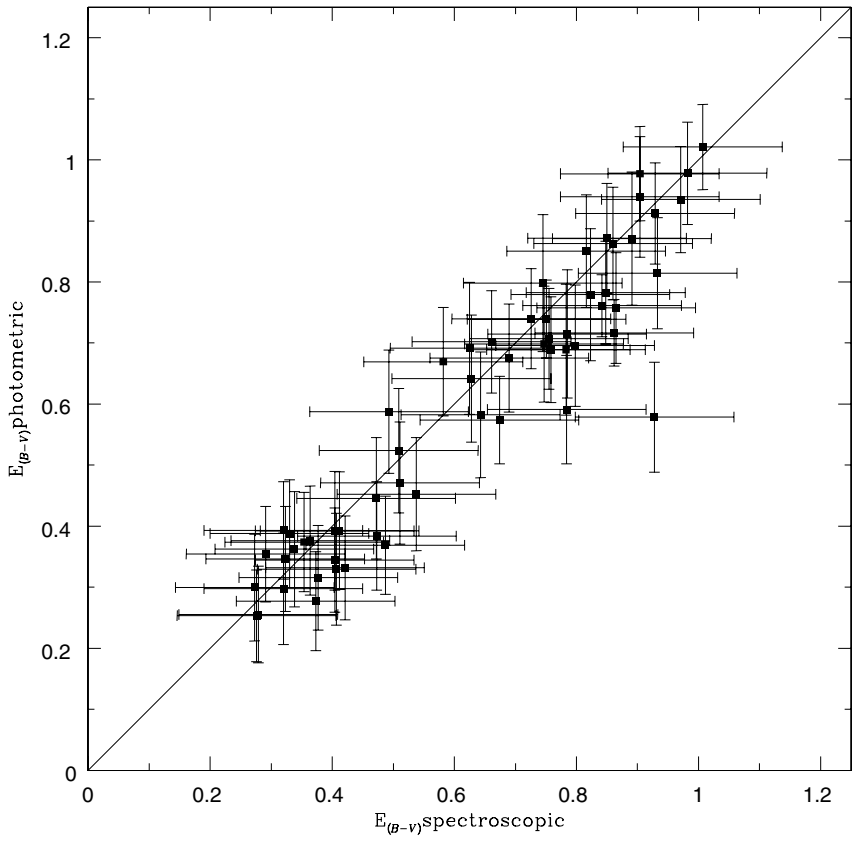

Fig. 17. Comparison of the colour excesses $(B-V)$ obtained using the combination of photometric and spectroscopic information of our stars in the $x$-axis and using only photometric with CHORIZOS code (Maíz-Apellániz 2004) in the $y$-axis. Both data sets have their respective errors.

\subsection{Distance}

In a previous paper (Corti et al. 2003) a mean distance modulus for the LS stars in the Bo 7 area was obtained using Eq. (4), where $M_{\mathrm{v}}$ (Schmidt-Kaler 1982) was estimated from the stars' spectral type and $A_{\mathrm{v}}$ was directly calculated from their intrin-

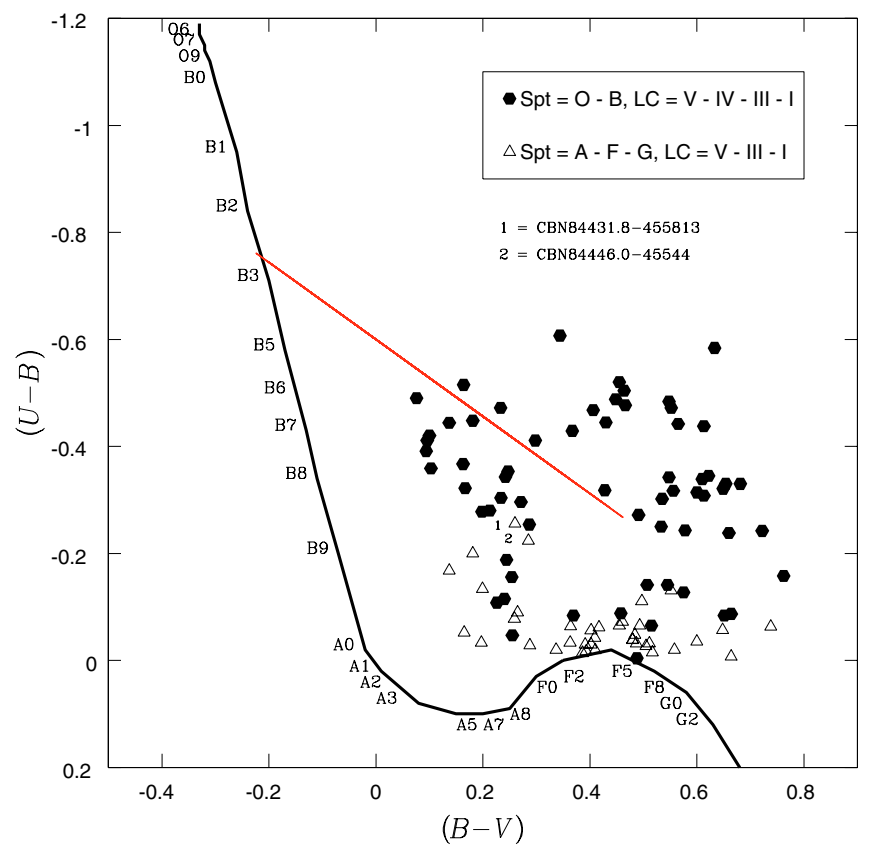

Fig. 18. Colour-colour diagram of the stars in the field of Bochum 7 with photometric and spectroscopic data. The sixty-three OB stars are plotted with filled hexagons and the other thirty-nine stars with spectral types between $\mathrm{A} 0$ and $\mathrm{G} 3$ are plotted with open triangles. The line represents the standard extinction curve for the $\mathrm{B} 3$ spectral types. The stars named CBN84431.8-455813 and CBN84446.0-45544 do not fall where expected according to their spectral types. They could be unresolved visual binaries.

sic colours (Schmidt-Kaler 1982), assuming a canonical relation between total and selective extinction. We can now extend the analysis to our larger sample and calculate the distance for the 63 OB spectral type stars. For these we employed the value of $R$ obtained by using the CHORIZOS code, mentioned previously, for each star. For those stars with emission lines, where the $R$ determination is unreliable, we replaced that value with $R=3.7 \pm 0.3$, obtained averaging all values of $R$ resultants for each star of the CHORIZOS code.

$V-M_{\mathrm{v}}=5 \times \log _{10}(\mathrm{~d})-5+A_{\mathrm{v}}$.

The value of mean distance obtained for the 63 stars is $4.2 \pm$ $2.1 \mathrm{kpc}$. The derived distances for the 63 stars are listed in Table 1. The analysis of this information combined with the kinematic of the proper motion, will be discussed in Paper II, in which we determine the membership to stellar groups.

\section{Summary}

Doing $U B V$ and $I R$ photometry and optical spectroscopy of the 63 OB spectral type stars found towards Bochum 7, we have:

- Obtained 6 stars with spectral type $O$ and 57 stars with spectral type B. Among these 63 stars, there are 11 giants and 52 dwarfs of main-sequence (MS) stars; 6 giants and 31 dwarfs of MS stars were discovered by us.

- Tested and confirmed that derivation of stellar parameters from our photometry (using CHORIZOS code) is highly reliable.

- Analysed the behaviour of interestellar extinction, finding that the spectral feature of the diffuse interstellar band $4428 \AA$ grows linearly with $E_{(B-V)}$ until $1 \mathrm{mag}$. For the rest 
of values of $E_{(B-V)}$, the feature of the DIB $4428 \AA$ is approximately constant.

- Estimated spectrophotometric distances to 63 stars, lying between 1.2 and $10.8 \mathrm{kpc}$ from the Sun.

Acknowledgements. We would like to thank the directors and staff of CTIO and CASLEO for the use of their facilities. We also acknowledge the use, at CASLEO, of CCD and the data acquisition system partly financed by US NSF Grant AST-90-15827 to Dr. R.M. Rich. This research has received financial support from IALP and research grant PIP2240, CONICET. This publication makes use of data products from the Two Micron All Sky Survey, which is a joint project of the University of Massachusetts and the Infrared Processing and Analysis Center California Institute of Technology, funded by the National Aeronautics and Space Administration and the National Science Foundation. M.C. thanks Gustavo Medina-Tanco for helpful suggestions and Nidia Morrell for encouraging her to join this project and providing some of the stars' spectrographs. We would also like to thank the referee, Jesús Maíz-Apellániz, whose comments and suggestions helped to improve the final version of this paper.

\section{References}

Cohen, M., Wheaton, W. A., \& Megeath, S. T. 2003, AJ, 126, 1090 Corti, M. 2005, Ph.D. Thesis. Universidad Nacional de La Plata, Argentina
Corti, M., Niemela, V., \& Morrell, N. 2003, A\&A, 405, 571

Cutri, R. M., Skrutskie, M. F., van Dyk, S., et al. 2003, 2MASS All Sky Catalog of point sources. The IRSA 2MASS All-Sky Point Source Catalog, NASA/IPAC Infrared Science Archive

http://irsa.ipac.caltech.edu/applications/Gator/

Herbig, G. H. 1975, ApJ, 196, 129

Kurucz, R. 2004, http: //kurucz .harvard.edu

Landolt, A. U. 1992, AJ, 104, 340

Lundstrom, I., \& Stenholm, B. 1984, A\&AS, 58, 163

Maíz-Apellániz, J. 2004, PASP, 116, 859

Maíz Apellániz, J. 2006, AJ, 131, 1184

Martins, F., Schaerer, D., \& Hillier, D. J. 2005, A\&A, 436, 1049

Moffat, A. F. J., \& Vogt, N. 1975, A\&AS, 20, 85

Reed, B. C. 2003, AJ, 125, 2531

Schmidt-Kaler, Th. 1982, in Landolt-Bornstein New Series, Group VI, Vol. 2b, ed. K. Schaifers, \& H. H. Voigt (Berlin: Springer-Verlag)

Snow, T. P., Zukowski, D., \& Massey, P. 2002, ApJ, 578, 877

Stephenson, C. B., \& Sanduleak, N. 1971, Publications of the Warner \& Swasey Observatory, 1,1

Sung, H., Bessell, M. S., Park, B.-G., \& Kang, Y. H. 1999, J. Korean Astron. Soc., 32, 109

van der Hucht, K. A. 2001, New Astron. Rev., 45, 135

Vijapurkar, J., \& Drilling, J. S. 1993, ApJS, 89, 293

Walborn, N. R., \& Fitzpatrick, E. L. 1990, PASP, 102, 379 
M. Corti et al.: OB stars in the field of Bochum 7. I., Online Material $p 1$

\section{Online Material}


M. Corti et al.: OB stars in the field of Bochum 7. I., Online Material $p 2$

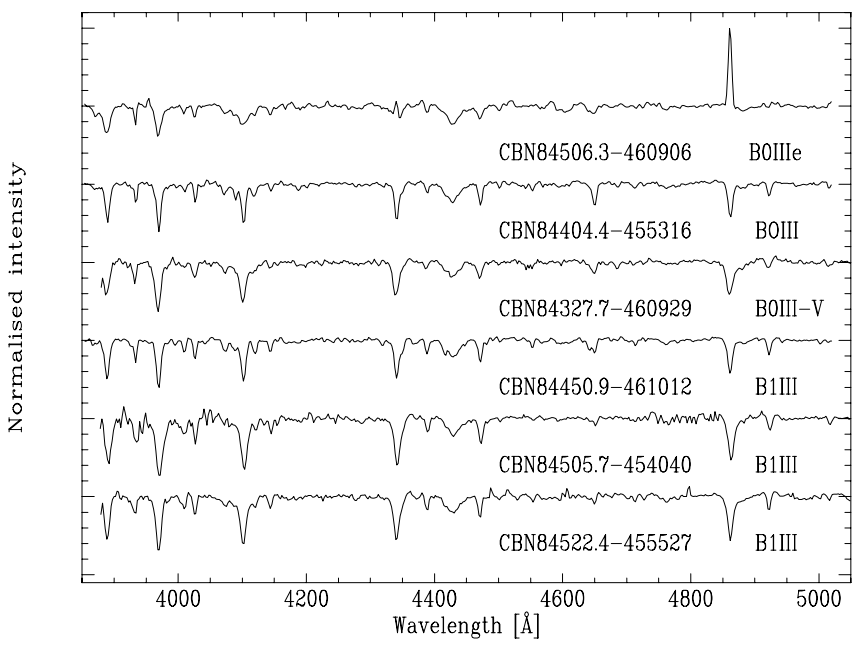

Fig. 6. This figure includes stars with B0-1III and B0IIIe spectral types in the region of Bochum 7. 1.

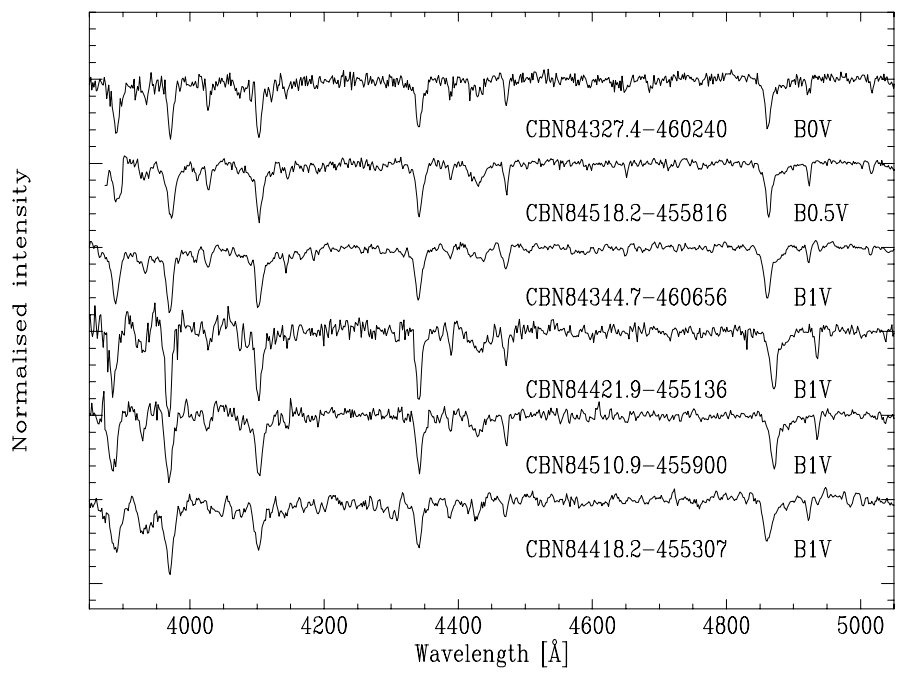

Fig. 7. This figure includes stars with B0-1V spectral types in the region of Bochum 7. 1.

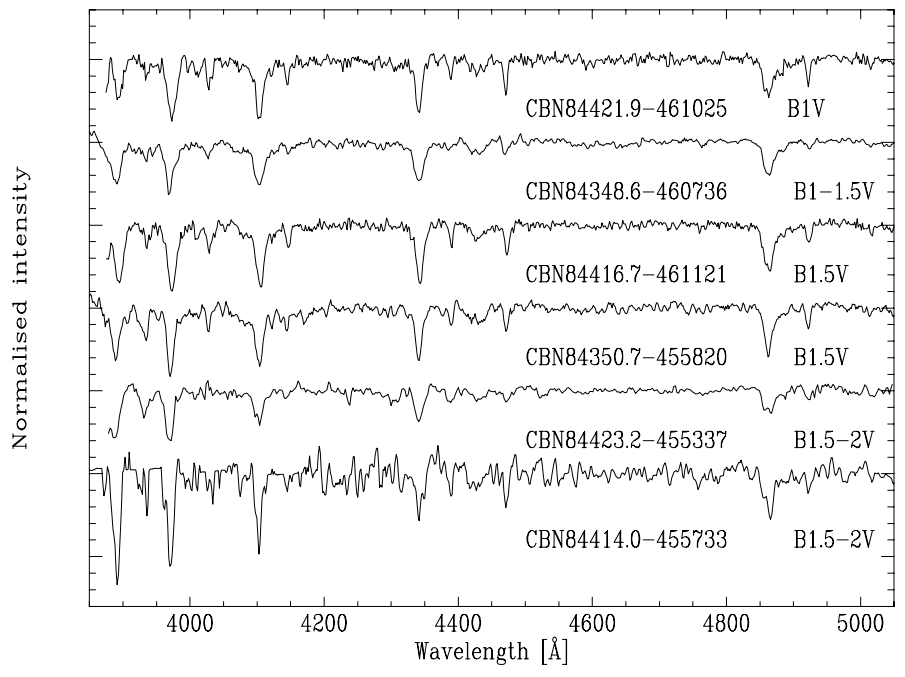

Fig. 8. This figure includes stars with B1-2V spectral types in the region of Bochum 7. 1.

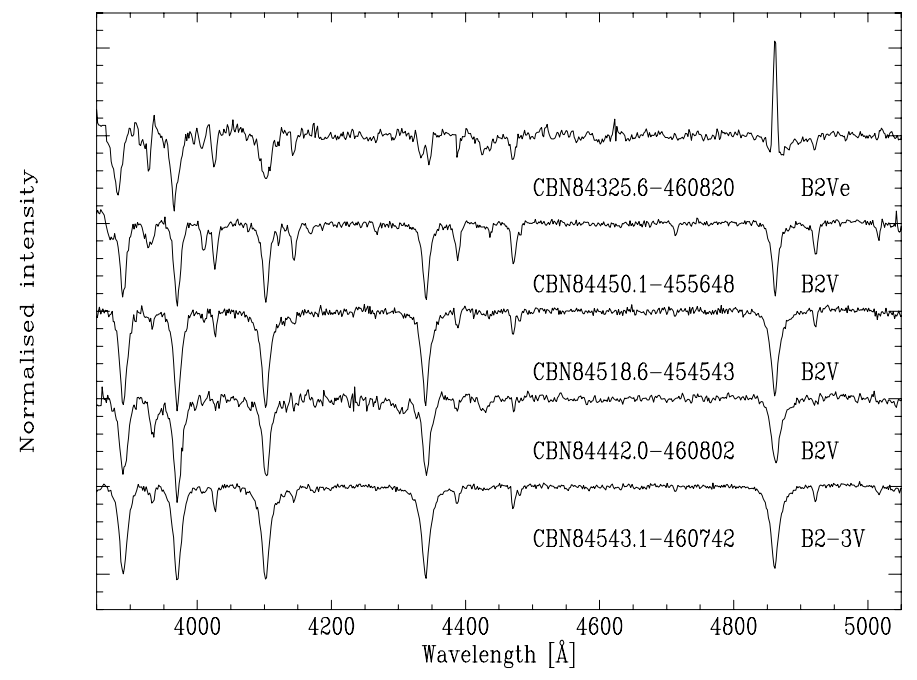

Fig. 9. This figure includes stars with B2-3V and B2Ve spectral types in the region of Bochum 7. 1.

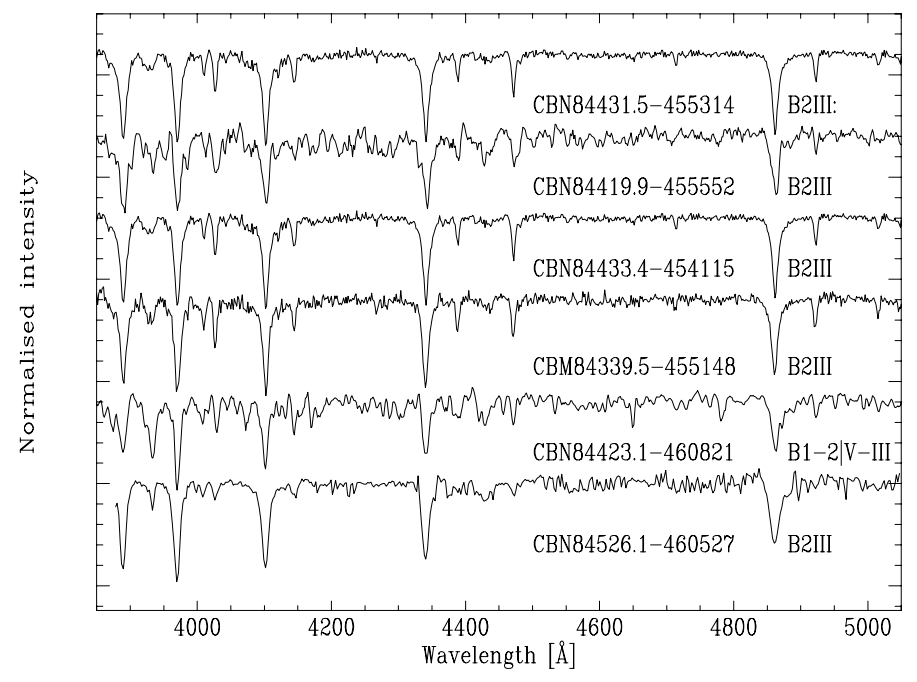

Fig. 10. This figure includes stars with B2III spectral types in the region of Bochum 7. 1.

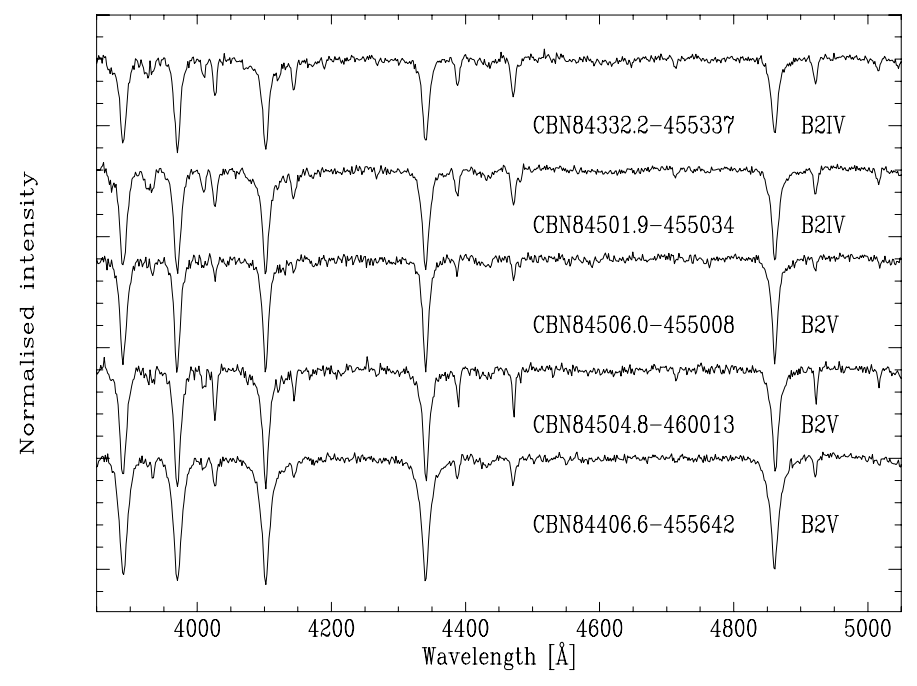

Fig. 11. This figure includes stars with B2IV-V spectral types in the region of Bochum 7. 1. 
M. Corti et al.: OB stars in the field of Bochum 7. I., Online Material $p 3$

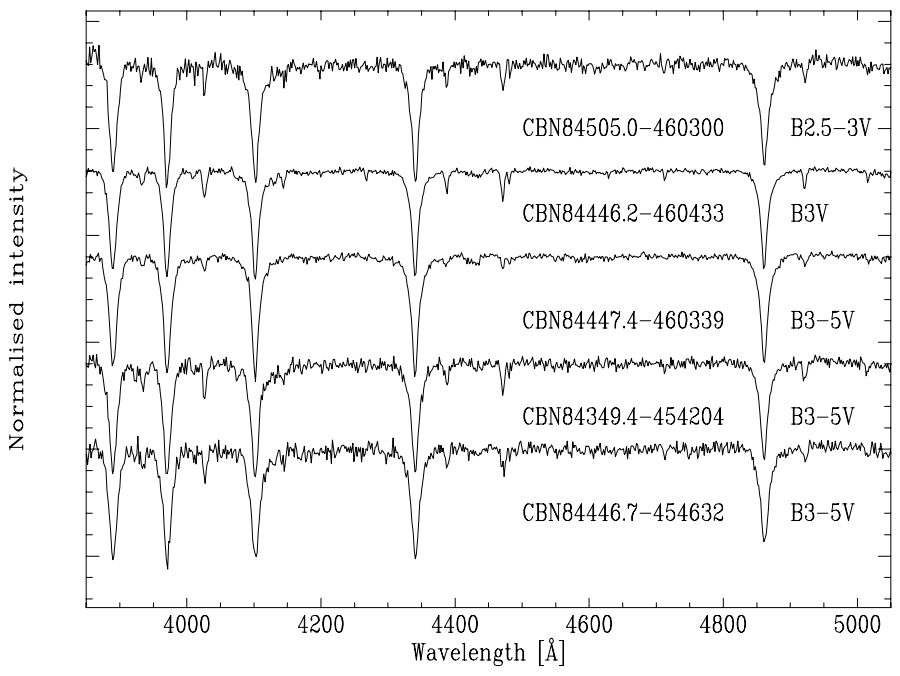

Fig. 12. This figure includes stars with $\mathrm{B} 3-5 \mathrm{~V}$ spectral types in the region of Bochum 7. 1.

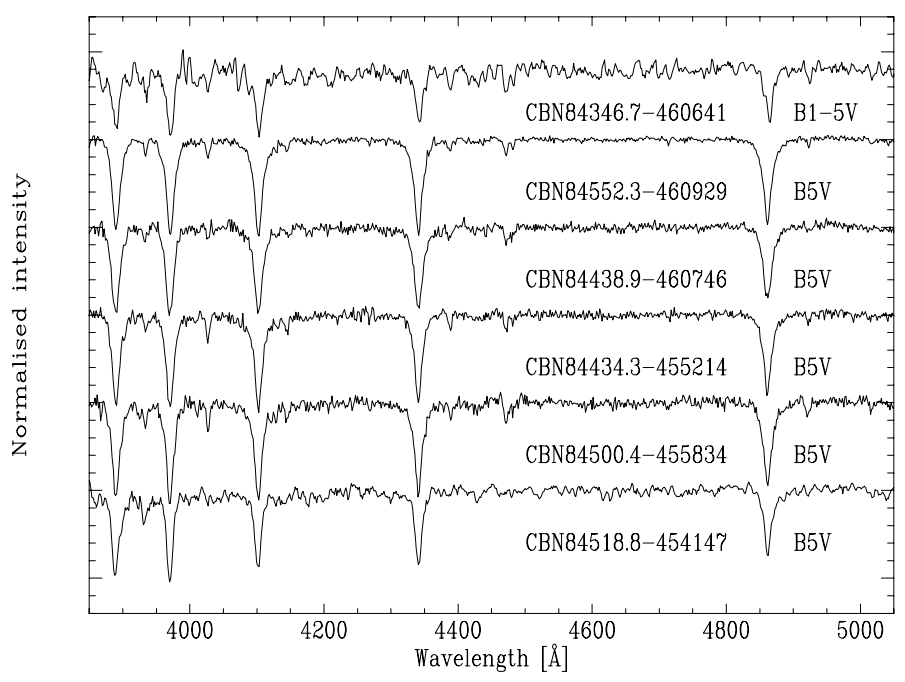

Fig. 13. This figure includes stars with B5V spectral types in the region of Bochum 7. 1.

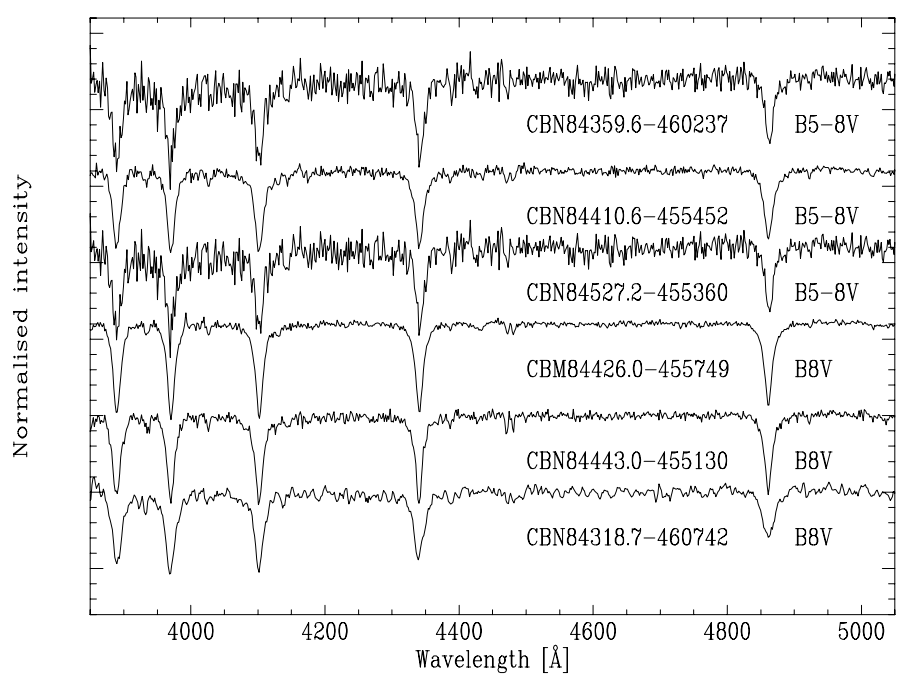

Fig. 14. This figure includes stars with B5-8V spectral types in the region of Bochum 7. 1.

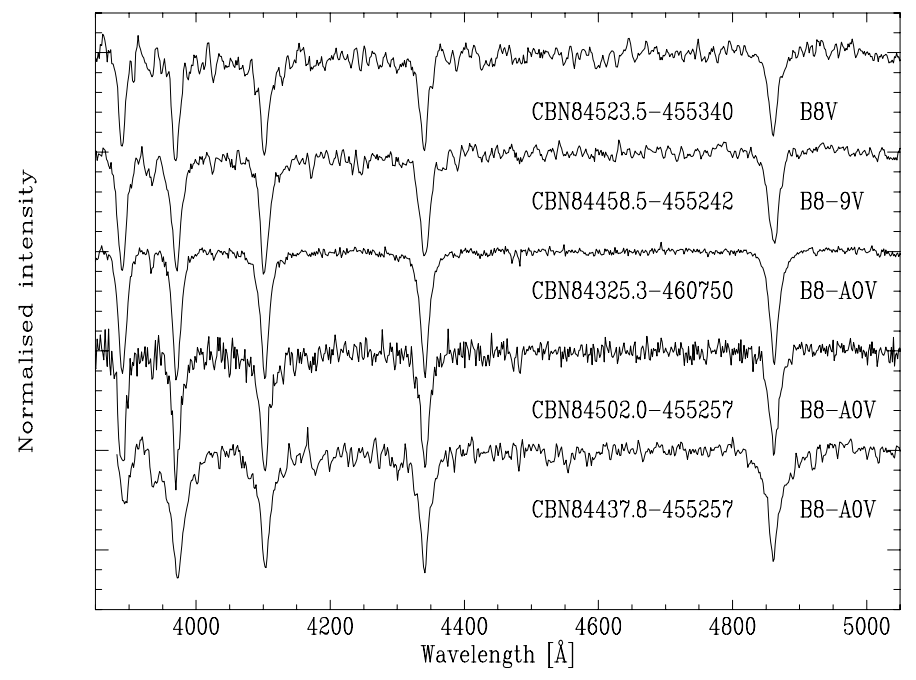

Fig. 15. This figure includes stars with $B 8-9 \mathrm{~V}$ and A0V spectral types in the region of Bochum 7. 1. 